\title{
Clinical Impact of Transesophageal Echocardiography in Patients with Stroke without Clinical Evidence of Cardiovascular Sources of Emboli
}

\author{
Solange Bernardes Tatani, Márcia Maiumi Fukujima, João Augusto Costa Lima, Luiz Darcy Cortez Ferreira, \\ Claudia G. Monaco G hefter, Gilmar Fernandes Prado, Zara Babayan, Lyamara A postólico de Azevedo
}

São Paulo, SP - Baltimore, MD

\begin{abstract}
Objective - The purpose of this study is to evaluate the impact of transeophageal echocardiography on management of patients at low-riskfor cardiogenic embolism to prevent new potential cardiovascular sources of emboli.

Methods - We studied 69 patients with ischemic stroke at low-riskfor cardiogenic embolism. Transeophageal echocardiography was performed to access: left atrium enlargement; communication or aneurysm of the interatrial septum; patent foramen ovale; spontaneous echo contrast or intracavitary thrombi; the presence of intraaortic atherosclerotic plaques or thrombi; significant valvar morphologic alteration or dysfunction; left ventricle enlargement, hypertrophy, or contractile abnormality. Transesophageal echocardiography altered clinical management, and we adopted anticoagulant therapy or another procedure apart from the use of acetylsalicylic acid.
\end{abstract}

Results - Transeophageal echocardiography detected at least one abnormality in 40 cases (58\%). Clinical conduct was adjusted after the performance of transesophageal echocardiography in 11 patients (15.9\%); anticoagulation was added in 10 cases and surgical correction in one patient.

Conclusion - Transeophageal echocardiography was a very useful tool in the secondary prevention for stroke in patients at low risk for cardiogenic embolism.

Key words: transeophageal echocardiography, stroke, embolism.

OMNI - Centro de Cardiologia Não Invasiva UNIFESP-Escola Paulista de Medicina, John Hopkins University - USA

Mailing address: Solange Bernardes Tatani - Rua Gil Fernandes, 183 - 04148-020 - São Paulo, SP - Brazil
The increasingly important role of stroke as a cause of mortality and disability is real. Statistics show that each year approximately 500,000 Americans experience a stroke and 147,800 die of stroke. Moreover, stroke accounts for roughly 2 million cases of permanent disability ${ }^{1}$. In Brazil, few epidemologic studies have been published on cerebrovascular diseases, even though they are the most frequent cause of death in the country ${ }^{2}$, with mortality rates in some Brazilian capitals 4 to 7 times higher than that in other international cities ${ }^{3}$.

Stroke may occur due to many causes, and a cardiovascular source of emboli is believed to be responsible for $30 \%$ of the cases. Among high-risk patients of embolismprone cardiac disorders are patients with mitral valve stenosis, atrial fibrillation (rheumatic and nonrheumatic), dilated myocardial disease, prosthetic valves, and recent myocardial infarction ${ }^{4}$.

In recent years, the use of transesophageal echocardiography has greatly contributed to the identification of other cardiac disorders that are being considered new potential causes of stroke, such as: patent foramen ovale ${ }^{5}$, atrial septal defect ${ }^{6}$, atrial septal aneurysm ${ }^{7}$, protruding aortic atheromatous plaque $(>4-\mathrm{mm} \text { thick })^{8,9}$, spontaneous echo contrast ${ }^{10}$, mitral valve strands ${ }^{11}$ and intracavitary thrombi (mainly in the left atrial appex).

The present study aimed at evaluating data obtained from transeophageal echocardiography in patients with ischemic stroke, who were not included in the therapy adopted in the high-risk cardiac group.

\section{Methods}

Sixty-nine patients were included in the study conducted between November ' 98 and September ' 99 . The ethics committee of the involved institutions approved the protocol-developed at John Hopkins University, and the patients gave written consent. The study protocol consisted of pa- 
tients with ischemic stroke who were admitted to the firstaid neurology clinic at the Escola Paulista de Medicina, regardless of age and sex. Neurologists performed the diagnosis and evaluation of patients, following a standard protocol, including a clinical examination and brain CT. Patients with atrial fibrillation, recent myocardial infarction (last six months), prosthetic cardiac valves, severe impairment of left ventricular function (IF $<20 \%$ ), formal contraindication for TEE, severely ill patients, and those receiving hospital care for more than 15 days were excluded from this study.

After hospital discharge, all patients underwent multiplane transeophageal echocardiography with a $5 \mathrm{mHz}$ transesophageal probe and with Vingmed echocardiographic System $\mathrm{V}$ (within 15 days). Images were recorded on videotape for later review by two observers. TEE examinations were performed after administration of topical anesthesia with an aerolized solution of lidocaine at $10 \%$ and intravenous sedation with midazolan $(1.5 \mathrm{mg} / \mathrm{ml})$ and meperidine $(50 \mathrm{mg} / \mathrm{ml})$. Contrast studies were performed by the rapid injection in the peripheral vein of microbubble solution $(6 \mathrm{ml}$ of isotonic saline $0.9 \%, 4 \mathrm{ml}$ of glucosis $50 \%$, and $1 \mathrm{ml}$ of air) at rest, during coughing, and during Valsalva maneuver. A comprehensive transeophageal echocardiography examination was performed with standardized scan planes. The following abnormalities were evaluated: left atrium enlargement, presence of masses, thrombi, or spontaneous contrast either inside the atrium or at the atrial apex or left ventricle. Patients were also evaluated for the following: interatrial septum aneurysm; patent foramen ovale, or any interatrial septal communication; fibrosis, mitral valve strands, calcifications, myxomatous degeneration, significant stenosis, or regurgitation of the mitral or aortic valve; enlargement, hypertrophy, left ventricle segmental or global dysfunction; and also atherosclerotic plaques or thrombi in the thoracic aorta.

Patent foramen ovale was diagnosed if more than three microbubbles were visualized in the left atrium within 5 cardiac cycles, following the opacification in the right atrium (Fig.1). Interatrial septal aneurysm was diagnosed when excessive expansion was observed $(>1.5 \mathrm{~cm}$ of bulging) at least $1.5 \mathrm{~cm}$ septum basis (do you mean that the bulge had to be measured as $1.5 \mathrm{~cm}$ at the base of septum?). Spontaneous echo contrast was characterized by smooth echoes with circular or spiral movement inside cardiac chambers. Mitral valve strands were defined as thin mobile filamentous projections attached to the atrial surface of mitral leflets or subvalvar apparatus. The left atrium was considered enlarged when it was $>40 \mathrm{~mm}$. The left ventricle was considered enlarged when diastolic diameter was $>50 \mathrm{~mm}$ and hypertrophic when diastolic thickness was $\geq 12 \mathrm{~mm}$; atherosclerotic plaques of in the ascending aorta, aortic arch, and descending aorta had their maximum thickness measured and were classified into 2 groups: those $<4 \mathrm{~mm}$ and those $\geq 4 \mathrm{~mm}$.

Immediately after the examination, a report with the results obtained was sent to the patient's personal physician. No events occurred during the echocardiography exam. A neurologist followed up patients within seven days after transeophageal echocardiography had been performed.

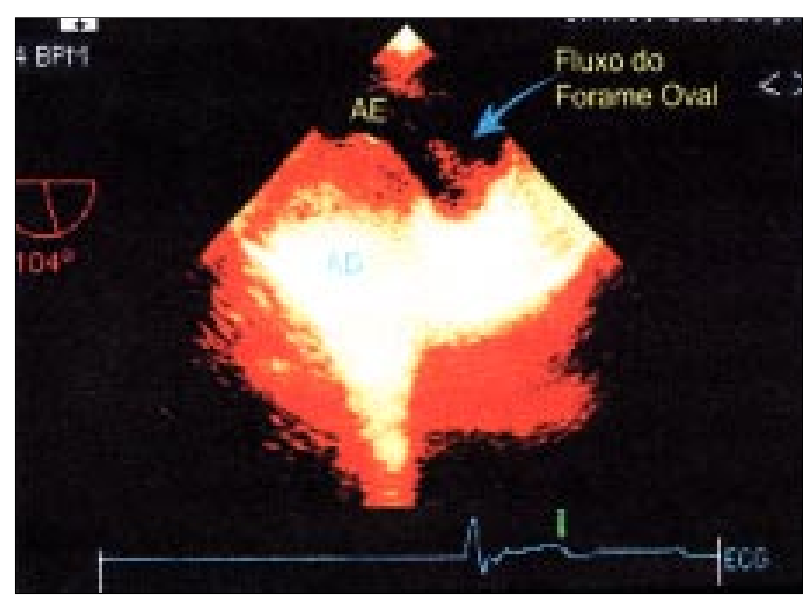

Fig. 1 - Transeophageal echocardiography with contrast through endovenous isotonic solution. We can notice right atrium opacificação (should this be "opacity") and the passage of microbubbles to the left atrium, during Valsalva maneuver.

\section{Results}

Sixty-nine patients with ages ranging from 12 to 90 years (mean age $63.4 \pm 14.1$ years); $52.2 \%$ were women. Of the patients, $44(63.8 \%)$ had hypertension, six $(8.7 \%)$ had diabetes, and two $(2.9 \%)$ had known coronary disease.

Left atrium enlargement occurred in 20 patients (29\%), left ventricle enlargement in 17 (24.6\%), myocardial hypertrophy in $30(43.5 \%)$, and mied or moderated left ventricle systolic impairment in $10(14.5 \%)$. Transeophageal study detected at least one potential cardiovascular source of emboli in 40 patients (58\%). Regarding interatrial septum, 15 (21.7\%) patients had patent foramen ovale (fig. 2), 7(10.1\%) had interatrial septum aneurysm, and one had interatrial communication. Of the patients who had interatrial septum aneurysm, four had associated patent foramen ovale. Intracardiac thrombus was observed in three patients $(4.3 \%)$; two thrombi were located in the left ventricle and one on the left atrial appendage. Spontaneous echo contrast was observed in 19 patients $(27.5 \%)$. Atherosclerotic plaques were detected in 54 patients $(78.3 \%)$, and in $63.8 \%$ of the cases those plaques were smaller than $4 \mathrm{~mm}$, and in $14.5 \%$ they were $4 \mathrm{~mm}$ or more thick. Mitral valve strands were not reported in any of the patients. Apart from these findings,

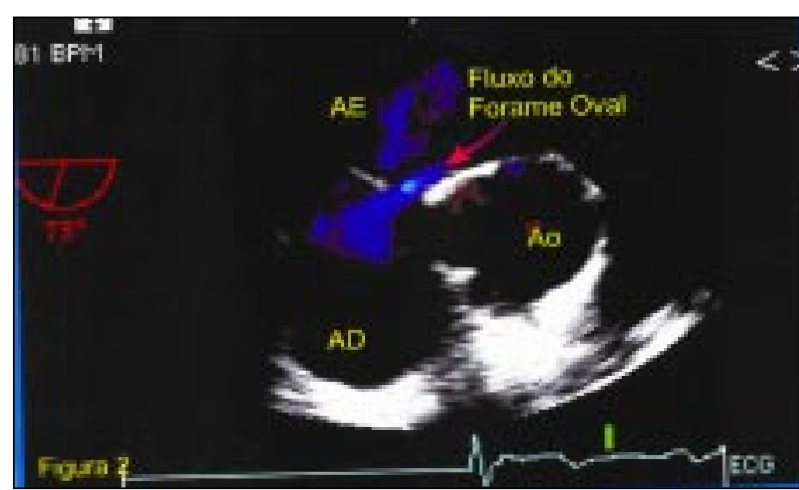

Fig. 2 - Transeophageal echocardiography showing flow through patent foramen ovale. 
we diagnosed in one patient, descending aorta dissection, mied mitral stenosis (with valvar area estimated at $1.6 \mathrm{~cm}^{2}$ ) and myxomatous degeneration of the mitral valve. In these cases, no clinical evidence existed to suggest diagnosis of these abnormalities, and another potential source of emboli existed in all cases.

Based on transeophageal study, therapeutics was changed in 11 patients $(15.9 \%)$. Ten patients received oral anticoagulant (warfarin), and the patient who had aortic dissection was sent to for surgical correction. Anticoagulant therapy was indicated in 3 cases of intracavitary thrombus, 2 cases of isolated patent foramen ovale, 3 cases of patent foramen ovale associated with interatrial septal aneurysm, patent foramen ovale, and one case of global and mied systolic left ventricle dysfunction. Oral anticoagulant was also indicated in one patient with mitral stenosis and patent foramen ovale, who had spontaneous echocardiography contrast inside left atrium. The other patients received acetylsalicylic acid.

\section{Discussion}

It is very important to identify the cause of ischemic stroke so that the therapeutics adopted can be defined, because the management of patients with cerebrovascular atherosclerotic stroke is substantially different from that of patients with ischemic stroke due to embolism. It is fundamental to secondary prevention of stroke because in $12 \%$ of these patients has a recurrent stroke.

Platelet antiaggregating drugs, especially acetylsalicylic acid, are the best option for patients with cerebrovascular atherosclerotic stroke in which platelets play an important role in the development of thrombi on occlusion of arteries, the vertebrobasilar system, or cerebral artery. On the other hand, if cerebral artery occlusion occurs from a cardiogenic thrombus made of mainly red cells and fibrin, anticoagulants should be used for both treatment and prevention.

Anticoagulant therapy prevents the development of new thrombi, thus preventing recurrence of ischemic stroke in patients with embolic diseases. Many studies have shown the benefits of anticoagulant therapy in patients with known embolic sources ${ }^{14,15}$ (especially in detecting intracardiac thrombi). No doubts exist concerning the need for indication of anticoagulant therapy in these cases.

Although it is important to define the cause of a stroke, the cause is generally presumed and seldom recognized. No consensus exists about the use of imaging strategies to be used in this investigation.

Transeophageal echocardiography has enabled identification of new potential sources of emboli, such as patent foramen ovale, interatrial septum aneurysm, spontaneous echocardiography contrast, and atheromatous aortic plaques in patients with ischemic stroke who are at low-risk for cardiac diseases. Transeophageal echocardiography is also more accurate in detecting intracardiac thrombus (especially in the left atrial appendage).

Patent foramen ovale has been related to paradoxical embolism, in which thrombi (intracavitary or peripheral) pass from the right to the left atrium through a patent foramen ovale. With transesophageal echocardiography, it is possible to visualize all the extension of the interatrial septum, and also to observe transeptal flow through foramen ovale. However, cases exist in which the orifice is very narrow with minimum transeptal flow and may not be noticed. To avoid false-negative results in such cases, we make use of contrast injection in the peripheral vein of agitated saline solution, at rest and after maneuvers, which causes an increase in pressure in the right side of the heart, thus determining the passage of contrast through patent foramen ovale. In 1988, Webster et al ${ }^{16}$ and Lechat et al ${ }^{17}$ reported a higher prevalence of patent foramen ovale in young patients with cryptogenic stroke (respectively, $40 \%$ and $50 \%$ ) when compared with patients without stroke $(10 \%$ and $15 \%$, respectively). After these studies, other studies using transeophageal echocardiography have proven the association of patent foramen ovale and stroke. Patent foramen ovale has also been associated with a higher rate of stroke recurrence. Mas et al ${ }^{18}$ found in a retrospective study a $4.4 \%$ annual rate of recurrence of events in patients with cryptogenic stroke and patent foramen ovale. Bogousslavsky et al ${ }^{19}$ performed a prospective study with stroke patients who were less than 60 years of age, in which they found a $1.9 \%$ annual rate of recurrence, in that, patent foramen ovale was one of the predictors of recurrence. However, few studies have been successful in proving venous thrombosis in patients with stroke and patent foramen ovale. Stöllberg et al ${ }^{20}$ after performing of transesophageal echocardiography documented venous thrombosis in 24 of the 42 patients with patent foramen ovale and with suspected paradoxical embolism. Nellensen et al ${ }^{21}$ detected through transeophageal echocardiography a thrombus overriding the interatrial septum in patients with deep venous thrombosis. However, other studies, such as the ones of Ranoux et al $^{22}$ and Gautier et al ${ }^{23}$, could not document venous thrombosis in patients with patent foramen ovale and stroke.

Correlation between interatrial septal aneurysm and embolism has been demonstrated ${ }^{7,24}$; however, the reason for embolism is yet unknown. Association with patent foramen ovale in such patients is frequent ${ }^{25}$, thus making them prone to paradoxical embolism.

The aorta is the most common site of atherosclerosis, surpassing the carotid and vertebral arteries. Risk of emboli increases according to the dimensions of atheromatous plaques and is higher in those $>4 \mathrm{~mm}$ thick; it is also higher in the presence of ulcerated plaques and when platelet deposits with thrombi are present, leading to occurrence of mobile debris on the surface ${ }^{8,9,26-, 28}$.

Spontaneous echo contrast is characterized by smooth echoes with slow circular or spiral movement, and its presence indicates blood stasis. In this condition, hemagglutination increases the probability of local embolization. Thrombi were visualized especially in atrial cavities, but also in ventricles and, sometimes even in the aorta. Spontaneous contrast has been considered a risk factor for embolism, 
which supports the findings of previous studies in the literature. A classic study by Chimowitz et al ${ }^{29}$ shows double embolic events in patients with mitral disease, who had spontaneous contrast on transesophageal echocardiography compared with those without spontaneous contrast.

Although transesophageal echocardiography has been proven to be a useful tool in the detection of new sources of emboli previously described, the routine use of this procedure in the patient with ischemic stroke is still controversial. The use of transeophageal echocardiography in risk stratification for new ischemic events was conducted at Johns Hopkins University. O'Brien and cols have published preliminary results of this study ${ }^{30}$, and have proven that it is possible to define the prognosis of patients with ischemic stroke using transesophageal echocardiography findings, which identify atherosclerotic aortic and spontaneous echo contrast as predictors of new event recurrence.
Furthermore, according to a study conducted by McNamara et $\mathrm{al}^{31}$, cost-effectiveness analysis of transeophageal echocardiography in the prevention of secondary stroke has been presented as the best cost-effectiveness method, when compared with other strategies of diagnosis and therapeutics frequently used in such patients.

In conclusion, we emphasize that absence of a cardiovascular source of emboli was the major criterion for the inclusion of patients in this study, taking into account that the procedure has changed clinical conduct significantly and has enabled the adoption of specific treatment, therefore, the importance of transesophageal echocardiography becoming clear in the evaluation of the heart as a source of emboli. To evaluate the impact of morbidity and mortality of these patients, the study continues with an increase of the sample, long-term follow-up of the patients, and control of the adopted treatment.

\section{References}

1. Wolf PA. An overview of the epidemiology of stroke. Stroke 1990; 21(suppl II): II-4-II-6.

2. Sistema de informações hospitalares do SUS (SIH/SUS). Mortalidade e morbidade hospitalar do SUS. Http://www.datasus.gov.br

3. Duncan BB, Schmidt MI, Polanczyk CA, Mengue SS. Altos coeficientes de mortalidade em população adulta brasileira: uma comparação internacional. Rev Assoc Med Bras 1992; 38: 138-44.

4. Cardiogenic brain embolism: the second report of the Cerebral Embolism Task Force. Arch Neurol 1989; 46: 727-43.

5. Di Tullio M, Sacco RL, Gopal A, MohrJP, Homma S. Patent foramen ovale as a risk factor for cryptogenic stroke. Ann Intern Med 1992; 117: 461-5.

6. Harvey JR, Teague SM, Anderson JL, Voyles WF, Thadani U. Clinically silent atrial septal defects with evidence of cerebral embolization. Ann Intern Med 1986; 105: 695-7.

7. Lucas C, GoullardL, Marchan M, et al. Higher prevalence of atrial septal aneurysms in patients with ischemic stroke of unknown cause. Acta Neurol Scand 1994; 89: 210-3.

8. Amarenco P, Cohen A, Tzourio C, et al. Atherosclerotic disease of the aortic arch and the risk of ischemic stroke. N Engl J Med 1994; 331: 1474-9.

9. Amarenco P, Duyckaerts C, Tzourio C, Hérnin D, Bousser M-G, Hauw JJ. The prevalence of ulcerated plaques in the aortic arch in patients with stroke. N Engl J Med 1992; 326: 221-5.

10. Briley DP, Giraud Gd, Beamer NB, et al. Spontaneous echo contrast and hemorheologic abnormalities in cerebrovascular disease. Stroke 1994; : 1564-9. (need volume\#)

11. Tice FD, Slivka AP, Walz ET, Orsinelli DA, Pearson AC. Mitral valve strands in patients with focal cerebral ischemia. Stroke 1996; 27: 1183-6.

12. Hart RG. Cardiogenic embolism to the brain. Lancet 1992; 339: 589-94.

13. Leonard AD, Newburg S. Cardioembolic stroke. J Neurosci Nurs 1992; 24: 69-76.

14. Baker RN, Broward JA, Fang HC. Anticoagulant therapy in cerebral infarction: report on cooperative study. Neurology 1962; 12: 823-35.

15. Report of the veterans administration cooperative study of atherosclerosis: an evaluation pf anticoagulation therapy in the treatment of cerebrovascular disease. Neurology 1964; 1: 132-8.

16. Webster MWI, Smith HJ, Sharpe DN, et al. Patent foramen ovale in young stroke patients. Lancet 1988; $2: 11-2$.

17. Lechat $P$, Mas JL, Lascault G, et al. Prevalence of patent foramen ovale in patients with stroke. N Engl J Med 1988; 318: 1148-52.

18. Mas JL, Zuber M. Recurrent cerebrovascular events in patients with patent foramen ovale or atrial septal aneurysm, or both, and cryptogenic stroke or TIA. Fren- ch Study Group on Patent Foramen Ovale and Atrial Septal Aneurysm. Am Heart J 1995; 140: 1083-8.

19. Bogousslavsky J, Garazi S, Jeanrenaud X, Aebischer N, Van Melle G. Stroke recurrence in patients with patent foramen ovale: The Lausanne Study. Neurology 1996; 46: 1301-5.

20. Stöllberg C, Slany J, Schuster I, Leitner H, Winkler WB, Karnik R. The prevalence of deep venous thrombosis in patients with suspected paradoxical embolism. Ann Intern Med 1993; 119: 461-5.

21. Nellensen U, Daniel WG, Matheis G, Oelert H, Depping K, Lichtlen PR. Impending paradoxical embolism from atrial thrombus: correct diagnosis by transesophageal echocardiographic and prevention by surgery. J Am Coll Cardiol 1985; 5: $1002-4$.

22. Ranoux D, Cohen A, Cabanes L, Amarenco P, Bousser MG, Mas JL. Patent foramen ovale: is stroke due to paradoxical embolism? Stroke 1993; 24: 331-4

23. Gautier JC, Dürr A, Koussa S, Lasclult G, Grosgogeat Y. Paradoxical cerebral embolism with a patent foramen ovale. Cerebrovasc Dis 1991; 1: 193-202.

24. Agmon Y, Khandheria BK, Meissner I, GentileF, Whisnant JP, Sicks JD. Frequency of atrial septal aneurysm in patients with cerebral ischemic events. Circulation 1999; 99: 1942-4.

25. Mügge A, Daniel WG, Angermann C, et al. Atrial septal aneurysm in adult patients. A multicenter study using transthoracic and transesophageal echocardiography. Circulation 1995; 91: 2785-92.

26. Karalis DG, Chandrasekaran K, Victor MF, Ross JJ, Mintz GS. Recognition and embolic potential of intraaortic atherosclerotic debris. J Am Coll Cardiol 1991; 17: 73-8.

27. Tunick PA, Rosenzweig BP, Katz ES, Freedberg RS, Perez JL, Kronzon I. High risk for vascular events in patients with protruding aortic atheromas: a prospective study. J Am Coll Cardiol 1994; 23: 1085-90.

28. Stone DA, Hawke MW, LaMonte M, et al. Ulcerated atherosclerotic plaques in the aorta are associated with cryptogenic stroke: a multiplane transesophageal echocardiographic study. Am Heart J 1995; 130: 105-8.

29. Chimowitz MB, DeGeorgia MA, Poole RM. Left atrial spontaneous echo contrast is highly associated with previous stroke in patients with atrial fibrillation or mitral stenosis. Stroke 1993; 24: 1015-9.

30 O'Brien PJ, Thiemann DR, McNamara RL, et al. Usefulness of transesophageal echocardiography in predicting mortality and morbidity in stroke patients without clinically known cardiac sources of embolus. Am J Cardiol 1998; 81:.

31. Mc Namara RL, Lima JAC, Whelton PK, Powe NR. Echocardiographic identification of cardiovascular sources of emboli to guide clinical management of stroke: a cost-effectiveness analysis. Ann Int Med 1997; 127: 775-87. 